\title{
A Smart Machine Learning Model for the Detection of Brain Hemorrhage Diagnosis Based Internet of Things in Smart Cities
}

\author{
Hang Chen, ${ }^{1}$ Sulaiman Khan $\mathbb{D}^{0},{ }^{2}$ Bo Kou, ${ }^{3}$ Shah Nazir $\mathbb{D}^{\circ},{ }^{2}$ Wei Liu $\mathbb{D},{ }^{4}$ and Anwar Hussain ${ }^{2}$ \\ ${ }^{1}$ Department of Information Service, Shaanxi Provincial People's Hospital, Xi'an, 710061, China \\ ${ }^{2}$ Department of Computer Science, University of Swabi, Ambar, Khyber Pakhtunkhwa, Pakistan \\ ${ }^{3}$ Department of Otorhinolaryngology-HeadઐNeck Surgery, The First Affiliated Hospital of Xi'an Jiaotong University, \\ Xi'an, 710061, China \\ ${ }^{4}$ Department of Vascular Surgery, The First Affiliated Hospital of Xi'an Jiaotong University, Xi'an, 710061, China
}

Correspondence should be addressed to Wei Liu; lizhang1988617@163.com

Received 5 July 2020; Revised 15 August 2020; Accepted 24 August 2020; Published 15 September 2020

Academic Editor: Atif Khan

Copyright (c) 2020 Hang Chen et al. This is an open access article distributed under the Creative Commons Attribution License, which permits unrestricted use, distribution, and reproduction in any medium, provided the original work is properly cited.

Generally, the emergence of Internet of Things enabled applications inspired the world during the last few years, providing stateof-the-art and novel-based solutions for different problems. This evolutionary field is mainly lead by wireless sensor network, radio frequency identification, and smart mobile technologies. Among others, the IoT plays a key role in the form of smart medical devices and wearables, with the ability to collect varied and longitudinal patient-generated health data, and at the same time also offering preliminary diagnosis options. In terms of efforts made for helping the patients using IoT-based solutions, experts exploit capabilities of the machine learning algorithms to provide efficient solutions in hemorrhage diagnosis. To reduce the death rates and propose accurate treatment, this paper presents a smart IoT-based application using machine learning algorithms for the human brain hemorrhage diagnosis. Based on the computerized tomography scan images for intracranial dataset, the support vector machine and feedforward neural network have been applied for the classification purposes. Overall, classification results of $80.67 \%$ and $86.7 \%$ are calculated for the support vector machine and feedforward neural network, respectively. It is concluded from the resultant analysis that the feedforward neural network outperforms in classifying intracranial images. The output generated from the classification tool gives information about the type of brain hemorrhage that ultimately helps in validating expert's diagnosis and is treated as a learning tool for trainee radiologists to minimize the errors in the available systems.

\section{Introduction}

Globally, most of the countries are facing a dramatic increase in the number of medical patients. This high rise in number restricts the patients in accessing the caregivers or primary doctors for quality treatment. In this modern technological age, improving the efficiency of biomedical and healthcare systems is the most challenging task. As highlighted by Redondi et al. [1], in fact, the current available procedures for patient care, supervision, monitoring, and management are executed by nursing staff manually. Absolutely, providing a quality treatment to patients with low cost, in short time period, and tackling the shortage of nursing staff are the key issues. During the past few years, the development of
Internet of Things (IoT) devices and wearable devices has improved quality of treatment by applying remote patient monitoring. Nowadays, IoT devices play a vital role in healthcare applications especially in detecting and diagnosing several kinds of diseases in smart cities, navigation system implementation [2], efficient traffic prioritization mechanism in the IoT devices [3], strengthening the Internet resource content, and managing a huge number of Internet connections using information centric network (ICN) $[4,5]$. Ahmad et al. [6] proposed the optimized clustering mechanism for vehicular ad hoc networks using genetic algorithms and honey bees model.

Brain hemorrhage is a type of stroke that is normally caused by an artery in the brain. The stroke bursts and bleeds 
in the surrounding tissues. The continuous bleeding from the concern tissues kills brain cells. In case of not accurately diagnosing and treated in time-sensitive procedure, then it will cause death or lifetime disability. The main causes of brain hemorrhage are regular use of alcohol, high blood pressure, smoking, etc., while heredity is also considered as a major factor in the context of brain hemorrhage. Computerized tomography (CT) scans are examined by the radiologists to predict intracranial hemorrhage (ICH) and locate affected regions. Additionally, hemorrhage is divided into five categories based on the bleeding tissues that are intraventricular hemorrhage, intraparenchymal hemorrhage, subarachnoid hemorrhage, epidural hemorrhage, and subdural hemorrhage. The medical specialists say that after traumatic brain injury (TBI), ICH occurs that leads to death or body paralysis for lifetime if clinically fails in accurate diagnosing and treatment processes [7].

This paper presents an IoT-based model for the brain hemorrhage detection based on support vector machine (SVM) and feedforward neural network (FNN). Rest of the paper is organized as follows: Section 2 indicates the literature and background information in association with the relevant primary studies. Similarly, Section 3 gives the detail about the proposed methodology. Section 4 provides the detail regarding the classification results generated by the classification techniques, followed by the conclusion in Section 5.

\section{Literature Review and Background Study}

Traumatic brain injury (TBI) is considered one of the main causes of death and disability in the United States of America (USA). It was estimated during the census 2013 that for all the injury deaths in USA, TBI contributes about 30\% [8]. After TBI accident intracranial injuries, fractures may arise that has the highest ratio of mortality [9]. Intracranial hemorrhage $(\mathrm{ICH})$ can cause a brain hemorrhage that is clinically considered the most severe case in treatment because if it is not treated accurately, it leads to the whole body paralysis or death. A CT scan machine is considered as one of the useful tools in case of emergency for the evaluation of TBI patients. Moreover, the CT scan machine captures multiple layers of the brain for diagnosing brain hemorrhage [10].

With the passage of time, several IoT-based healthcare devices are developed that provide medical facilities for patients without interruptions or long waits in the hospital or even some of the devices provide diagnosing facilities at door steps. Nazir [11] presented a systematic literature review on IoT-based applications in healthcare using the effects of mobile computing. Onasanya and Elshakankiri [12] presented an IoT-based cancer care and business analytics services system. Cappon [13] proposed an IoTbased device for continuous glucose monitoring. This is a wearable device. Ullah [14] presented a review article on wireless body area networks (WBAN) to analyze the available literature and address the challenges based on the current environment and needs and traffic priority awareness in the Internet for IoT devices [15]. Kodali and
Sarjerao [16] suggested IoT-based application for urban climate monitoring. This application is developed using Raspberry Pi. Some of the healthcare devices are developed using the smart phone devices. Table 1 represents a few of the IoT-based healthcare applications using smart phones.

Neural network is prominent in classifying the images with high classification rates, and that is why deep neural networks are followed in different image classification problems. Likewise, $\mathrm{NN}$ has the ability to automate the segmentation process of ICH images to diagnose and detect brain hemorrhage [17]. An automated ICH screen tool will certainly help the radiologist to find out the brain hemorrhage even with little experience in the proposed field or without the presence of experts in the emergency rooms that is most common in the developing countries. The emergence of IoT devices has evolved the world by automating all the machinery work using sensors and limited human interaction with devices (need of technicians). This emerging field has revolutionized the healthcare system in smart cities worldwide where the patients can access quality treatment at door step. Different techniques are proposed for telehealth purposes based on IoT devices. For example, Abdelaziz [18] proposed a machine learning model based on linear regression and neural network for chronic kidney disease diagnosis and prediction.

Al-Majeed et al. [19] proposed a home telehealth system based on IoT devices. Dwivedi et al. [20] proposed block chain technology for data privacy in IoT devices. Firouzi [21] suggested smart sensors for healthcare applications. Hassanalieragh [22] addressed the current issues faced by the IoT devices and presented solutions in order to handle these crucial issues. Jabbar [23] developed an IoT semantic-based interoperability model (IoT-SIM) to develop semantic interoperability among heterogeneous IoT devices in the healthcare domain. Maktoubian and Ansari proposed an integrity monitoring system for ensuring security and privacy of healthcare IoT devices [24]. Mutlag et al. [25] presented a systematic literature review for fog computing in the healthcare IoT devices. Shakeel [26] put forward a deep Q-network for restricting malware attacks in healthcare IoT devices.

\subsection{Regional-Based Market Share of IoT in Healthcare} (2014-2022). Worldwide, in the past few years, the encapsulation of technology in healthcare devices immensely increased. For example, the IoT and data analytics are gaining popularity particularly in eHealth and $\mathrm{m}$-Health services for the next generation. During the emergence phase, they provided new challenges in healthcare systems because they need highly accurate, efficient, safe, consistent, flexible, and energy efficient solutions. The IoT in healthcare covers the markets of health devices, health software, and providence of high-quality health services. IoT devices are implemented in the smart cities in the developed countries which prevail in the developing countries.

Figure 1 represents a regional-based IoT market share. During the census 2014, the market analyst, Grand View Research, reported that North America dominated the 
TABLE 1: Smart phone-based healthcare applications.

\begin{tabular}{|c|c|}
\hline Mobile applications & Descriptions \\
\hline Eye Care Plus & Using the eye health information, this application helps in improving vision abilities naturally \\
\hline $\begin{array}{l}\text { Blood Pressure } \\
\text { Watch }\end{array}$ & A wearable device used in monitoring, collecting, and storing the records of blood pressure data \\
\hline Google Fit & sensors, it tracks running, walking, or cycling activities automatically \\
\hline Noor & fitness \\
\hline Healthy Child & This helps in finding a baby doctor at nearer locations and requesting for quick responses \\
\hline Health Assis & $\begin{array}{c}\text { This application stores the records of different health parameters like blood pressure, weight, body temperature, and } \\
\text { other physical activities }\end{array}$ \\
\hline
\end{tabular}
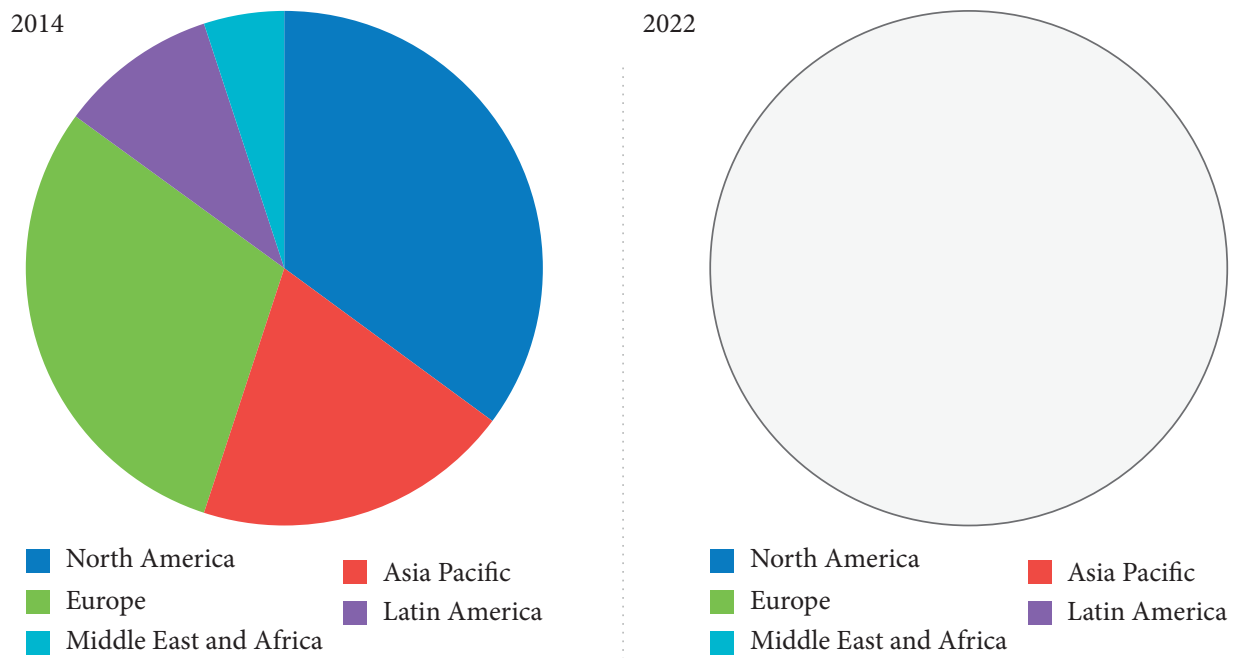

FIgURE 1: Regional-based IoT market share during 2014-2022.

overall market in healthcare IoT services [2]. Key factors reported for this high contribution are due to the government favors for providing high budgets to the cyber security budget and mandatory implementation of electronic health record (EHR) systems since 2015. The private funding and government interest in the IoT services are the key accelerating factors in this dominance in North America.

Contrary to this, no such government or private interest is shown in Asia Pacific, Latin America, or Middle East and Africa regions. These specific countries share about $20 \%$, $10 \%$, and $5 \%$, respectively, as shown in Figure 1 . This low share also reflects the quality of treatment and deprival of quality health in these regions.

2.2. Market Estimates (2014-2025). The IoT in the healthcare market is categorized on the basis of region, end-user, components, application, and connectivity technologies. However, the market is categorized based on system and software applications, medical devices, and the type of services it provides. Several types of medical devices facilitated with IoT technology are stationary devices, wearable devices, and implanted medical devices. The connected devices are those devices that consume scarce resources. These types of devices are designed to improve the quality of care and treatment that result in better clinical outcomes. Moreover, on account of the high demand for effective care in case of pediatric or aged persons, eHealth and m-Health services, personal health and management, and chronic disease management are some of the key factors that are taken into consideration to boost the healthcare device market with the application of IoT technology.

However, the market is also segmented based on system and software applications that consists of the device and network security and management, remote device management, data analytics, and network bandwidth management. Based on the services, the IoT is classified into training, educating, consulting, integration services, and maintenance services. The Grand View Research analyst reported that during the period 2014-2025, the market growth of IoT devices will be more than $\$ 300$ billion as shown in Figure 2. This budget covers all the categories of IoT-based medical devices, system, and software and services. Government enterprises also help in promoting these IoT-based applications for personal eHealth services.

From Figure 2, it is concluded that with the passage of time, the growth in IoT-based medical devices increases due to its high-quality treatment and limited user interaction.

2.3. IoT-Based Healthcare Model. Figure 3 represents a conventional diagram of the IoT-based healthcare model. This model mainly consists of three components [27]: (1) the body area sensor network, (2) smart devices connected through the Internet also known as fog layer or also known as local access networks, and (3) cloud and big data support. 


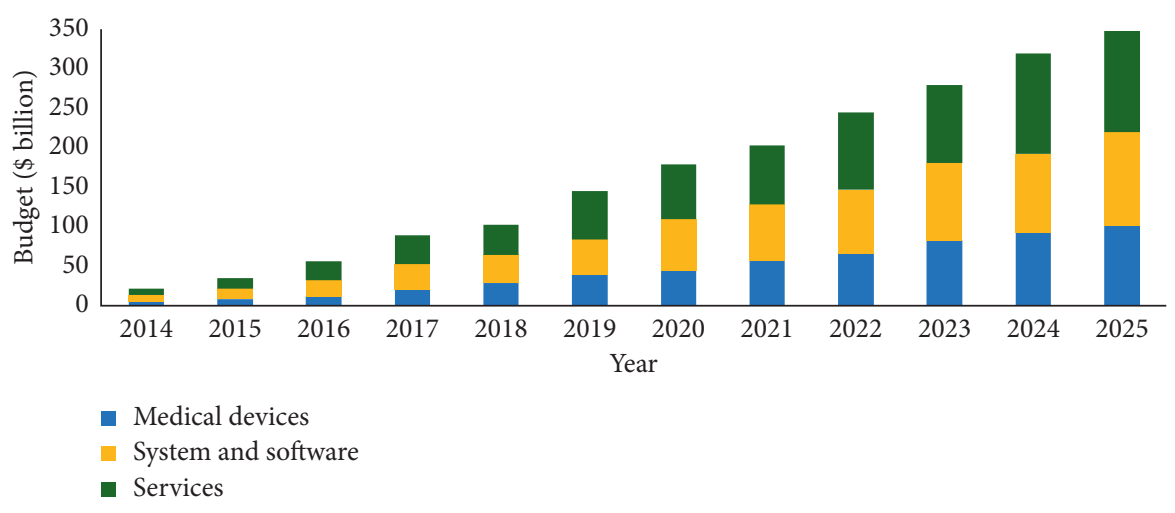

Figure 2: North America IoT in healthcare market (2014-2025).

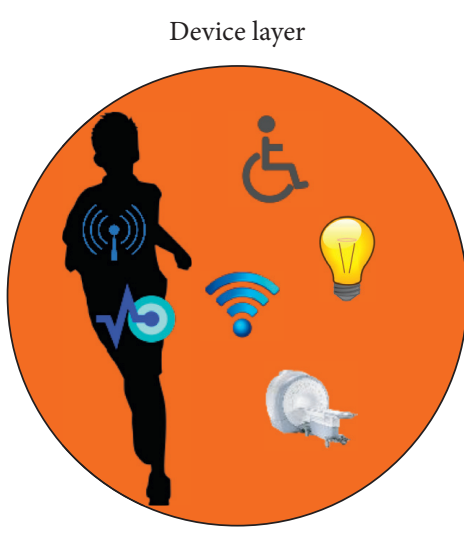

(i) Sleep monitoring

(ii) Vital signs

(iii) Fall detection and activities

(iv) Environmental info

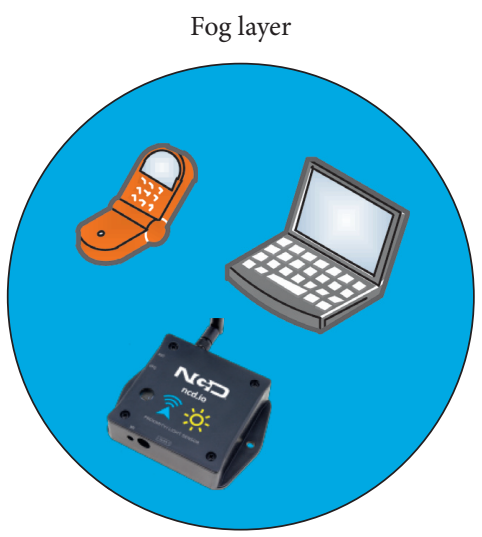

(i) Data processing

(ii) Local notification

(iii) Data filtering and mining

(iv) Protocol coversion

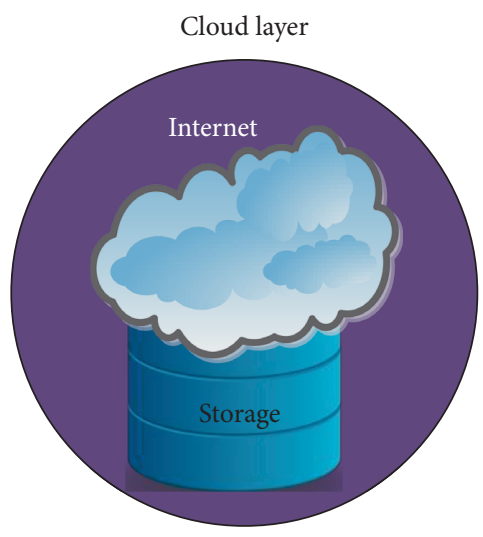

(i) Data analytics

(ii) Data storage

(iii) Decision-making

(iv) Medical caregivers inference

FIgURE 3: Conventional IoT healthcare model.

The third component in the conventional architecture provides services to different stockholders. Data generated from the sensors are visible to doctors, families, and other authorized parties to access reports about the patient from anywhere through the Internet. This helps in assisting healthcare workers through intelligent decision-making tools [28].

In the conventional model given in Figure 3, smart eHealth gateways exist for supporting multiple communication protocols that ultimately work as a bridging point between local network and sensor networks. Using this bridging point, it receives data from different subnetworks, performs protocol conversion, and provides other higherlevel services such as data mining, filtering, aggression, local notification, and dimensionality reduction.

\section{Proposed Methodology}

The Internet of Things using mobile computing has a lot of applications in the field of healthcare which enable it more reliable, providing quality treatment. Some research studies describe how the healthcare is affected using mobile computing, the security of IoT devices in healthcare using mobile computing, and application of the IoT in healthcare systems [11]. Figure 4 shows the fluctuation and rising of the IoT-based research in healthcare using mobile computing in yearly distribution of the studies in the field.

Mobile health (m-Health) provides a massive support by extending the abilities and functionalities of the IoT devices in healthcare. The m-Health devices are directly connected to the IoT server for specific operations like blood pressure counting, level of sugar in blood, and heart rate. List of devices and their functionalities based on the IoT and healthcare are represented in Table 2.

Figure 5 represents the annual trends in the m-Health. From the figure, it is concluded that with the passage of time, the research work in the proposed field increases because of its high applicability in the healthcare fields.

From the above discussions, it is concluded that a lot of work is reported on the development of IoT devices for different types of diseases like blood sugar level calculation, pulse rate calculation, and speed calculation. The proposed research work presents a smart IoT-based brain hemorrhage detection system using machine learning approach. 


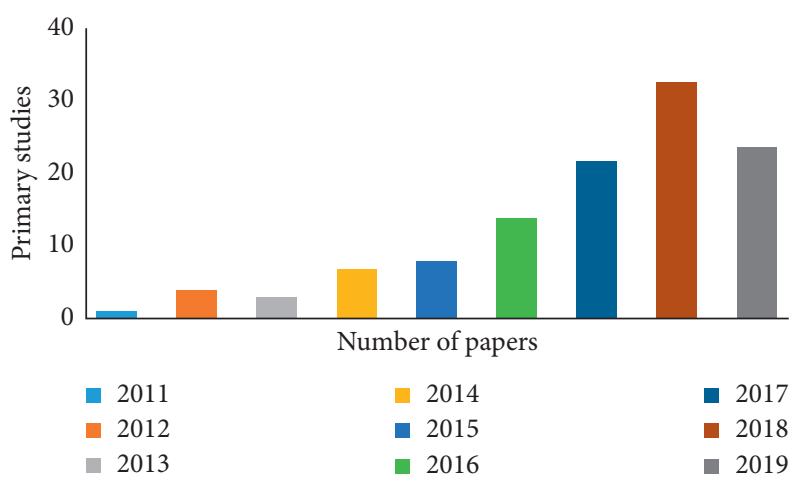

Figure 4: Primary studies reported annually.

TABle 2: Description of m-Health devices.

\begin{tabular}{lr}
\hline m-Health devices & Purpose \\
\hline $\begin{array}{l}\text { iBGSta } \\
\text { TICKR }\end{array}$ & Used in measuring the heart rates of the patient. This device calculates heart rates by strapping around the chest. \\
$\begin{array}{l}\text { FitBit surge } \\
\text { Forerunner 920XT }\end{array}$ & A measuring device that calculates sleeping patterns, heart rate, and loss of calories during a physical exercise. \\
& It keeps records of swimming stroke counter, step counter, and calorie calculation.
\end{tabular}

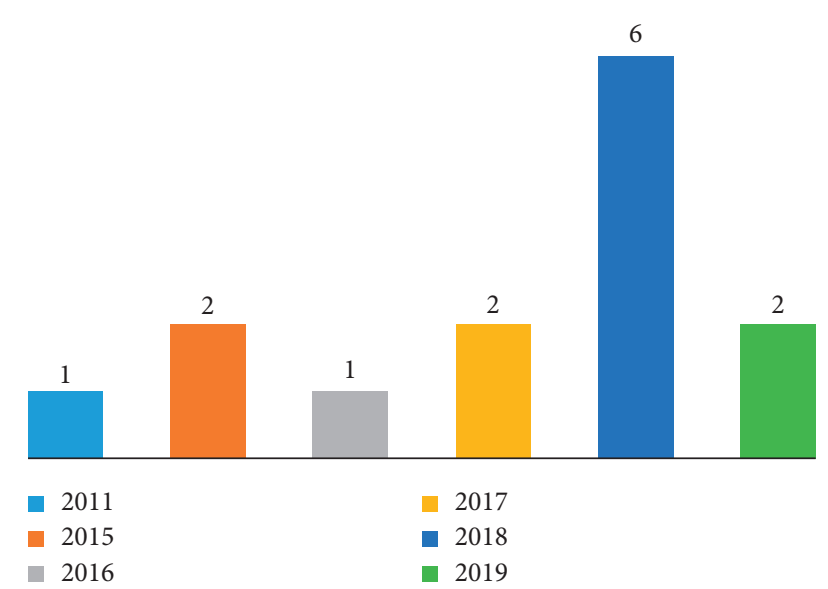

Figure 5: Trends in m-Health.

The entire process of the proposed research work is shown in Figure 6. It contains a CT scan machine that is used for capturing multilayer scans of the patient in case of brain hemorrhage. A Wi-Fi sensor is fixed in between the CT scanner and Arduino board. A number of components are used for the efficient communication among IoT devices. Description of these components is explained in the upcoming section.

(i) Sensors: in the proposed research work, two types of sensors are used for sensing data. These sensors are used for collecting CT scan images of the CT scanner machine, and after converting these images to electrical signals, they are stored on the server for medical purposes.

(a) Complementary metal oxide semiconductor (CMOS) sensor: these sensors are used for acquiring patient's images from the CT scanner machine, and they are converted to electrical signals for digital processing. These signals are fed to the Arduino UNO board for further processing.

(b) ESP8266 Wi-Fi module: this Wi-Fi module is used for posting data from the Arduino board to the web server. Arduino and ESP8266's Vcc and GND pins are directly connected to the power supply. Transmitter (Tx) and Receiver (Rx) pins of ESP8266 are directly connected to the Arduino board.

(ii) Classification techniques: the support vector machine and feedforward neural network are selected for classifying the CT scan images to diagnose the brain hemorrhage. Based on the classification results (also known as medical reports), the caretakers suggest medicines for the patients.

(iii) Mobile application: a mobile application is developed for both the doctor and the patient via which both can communicate. The report is automatically attached in Gmail, and the details of the patient are added automatically in the mail content. This report is transmitted to the physician. The physician sends the report and the corresponding medicines to the patient.

(iv) Main circuit board: Arduino UNO is selected for the overall circuit control purposes because of its low cost, easy to use and implement, and high applicability in different environments.

All the steps mentioned in Figure 6 are discussed in detail below.

3.1. Dataset. For the proposed research work, the dataset is collected from UCI machine learning dataset repository. The dataset name is "intracranial brain hemorrhage dataset" 


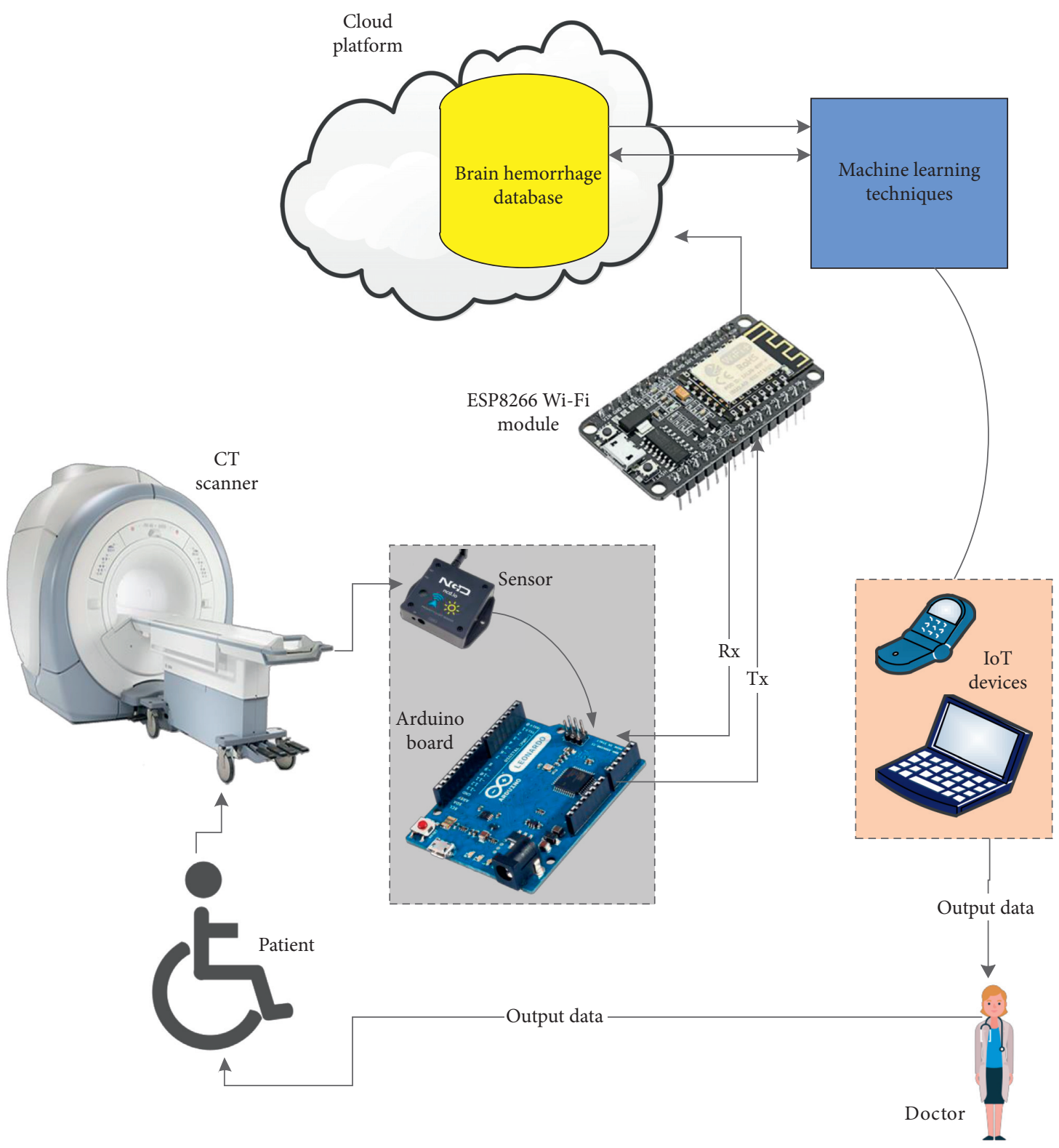

Figure 6: Proposed methodology.

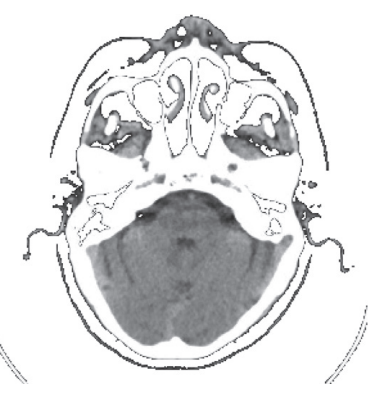

(a)

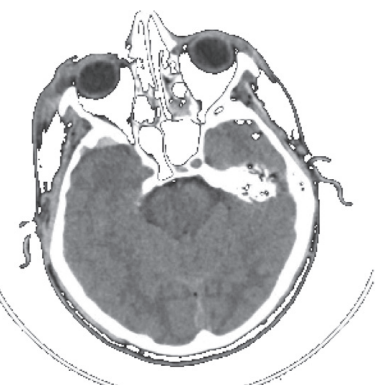

(b)
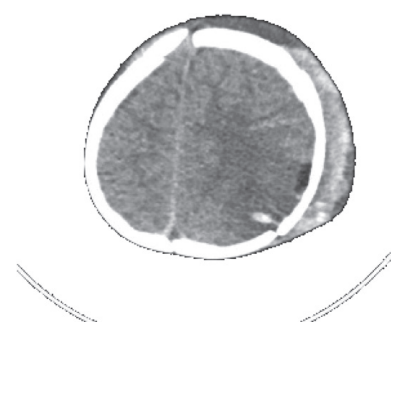

(c)

FIGURE 7: Brain hemorrhage CT scan images: (a) normal, (b) epidural hemorrhage, and (c) intraparenchymal hemorrhage. 
which has the following types: intraparenchymal, epidural, subarachnoid, intraventricular, and subdural [7]. It consists of $82 \mathrm{CT}$ scans collected from 36 different patients where 46 of the patients are males and 36 are females. Figure 7 shows some of the brain hemorrhage CT scan images.

3.2. Support Vector Machine. The support vector machine (SVM) is a binary classification technique followed in classifying different types of machine learning and pattern recognition problems. It is developed by Cortes and Vapnik $[29,30]$. It is followed for resolving many linear and nonlinear classification problems. Due to its binary classification nature, it is good in finding optimal hyperplane or decision boundary based on the feature map as shown in Figure 1:

$$
f(x)=W^{T} \theta \mathrm{x}+b,
$$

where $w$ and $b \in R$, while $\theta(x)$ is a feature map used in classification.

Since the problem consists of five classes, namely, intraparenchymal, epidural, subarachnoid, intraventricular, and subdural, SVM fails in classifying multiclass problems because it is a binary classifier, so in our case, we have followed the concept of one vs many approach as shown in Figure 8. In this approach, one class is selected as a base class and the rest of the classes are compared with that, then the second one, and so on.

The results of the proposed support vector machine classifier are shown in Figure 9. In this figure, the right-side numerals represent the calculated accuracy results, while the left-side shows different brain hemorrhage types. Overall accuracy result of $80.67 \%$ is calculated for SVM.

3.3. Neural Network. In the proposed research work, an attempt has been made using feedforward neural network for classification of brain hemorrhage CT scan images. From 1960s, the field of classification and recognition paced up, and the researchers developed different deep and shallow architectures for the automatic recognition purposes. The shallow architectures consist of SVM, $k$-nearest neighbors, Naïve Bayes, random forest decision, tress, and fuzzy logic, while the deep architectures consist of the architectures that work on a number of hidden layers. These hidden layer neurons automatically extract features and provide output based on the recognition/classification problems. The deep architectures comprise convolution neural network $(\mathrm{CNN})$, binary long short-term memory (BLSTM), artificial neural network (ANN), feedforward neural network (FNN), backpropagation neural network (BPNN), and many more.

After 2014, the neural networks, or in other words deep networks, are followed because of calculating high accuracy results for both classification and recognition problems. The neural networks are developed based on the human nervous system. It consists of neurons that accept inputs, process that data, and provide output patterns according to the data provided to the network. Due to their automatic feature extraction capabilities, mostly hidden architectures are

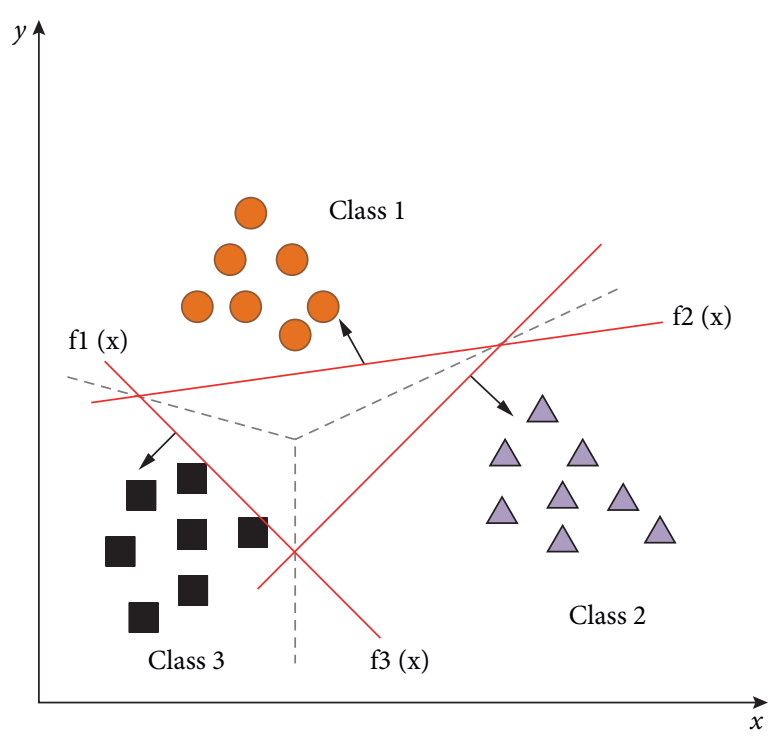

Figure 8: One vs many approach of the SVM technique.

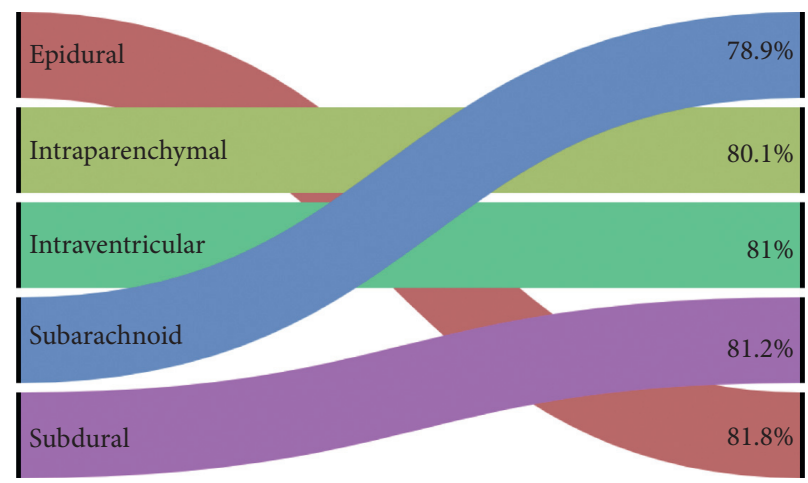

FiguRE 9: SVM classification results.

followed after 2014 [31]. In this research work, we implemented the feedforward neural network. A generic model of FNN is shown in Figure 10 that consists of three layers: one input layer, one hidden layer, and the output layer. The number of hidden layers varies.

In our case, a feedforward neural network has been developed that consists of 5 hidden layers. The output is generated based on varying training sets and test sets as shown in Figure 11. It is evident from Figure 11 that when the training set increases, the time increases and ultimately the accuracy rate also increases. In our case, feedforward neural network performs very well compared to the support vector machine because the neural networks are prominent in classifying images. Overall, an accuracy rate of $86.7 \%$ is calculated for the proposed feedforward network.

Figure 10 represents the accuracy of the proposed IoTbased device after applying the feedforward neural network for the identification of brain hemorrhage. From Figure 12, it was concluded that the FNN outperforms compared to SVM in the diagnosing process.

Figure 13 represents the model loss for the proposed IoT application. 


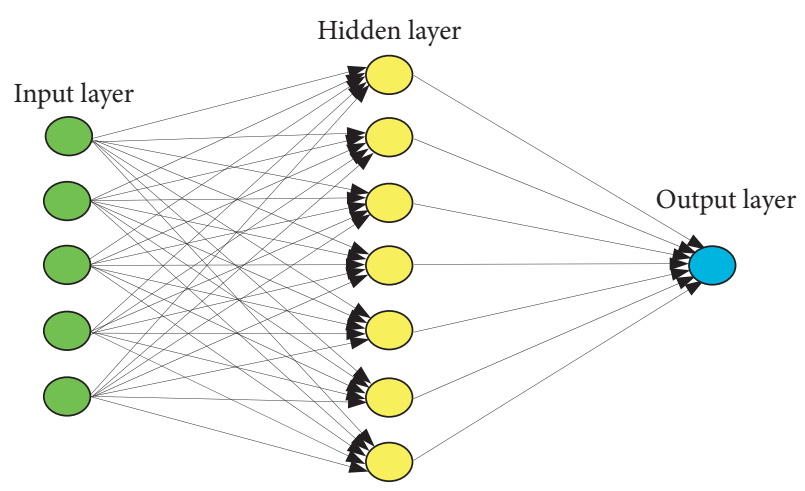

Figure 10: Generalized feedforward neural network.

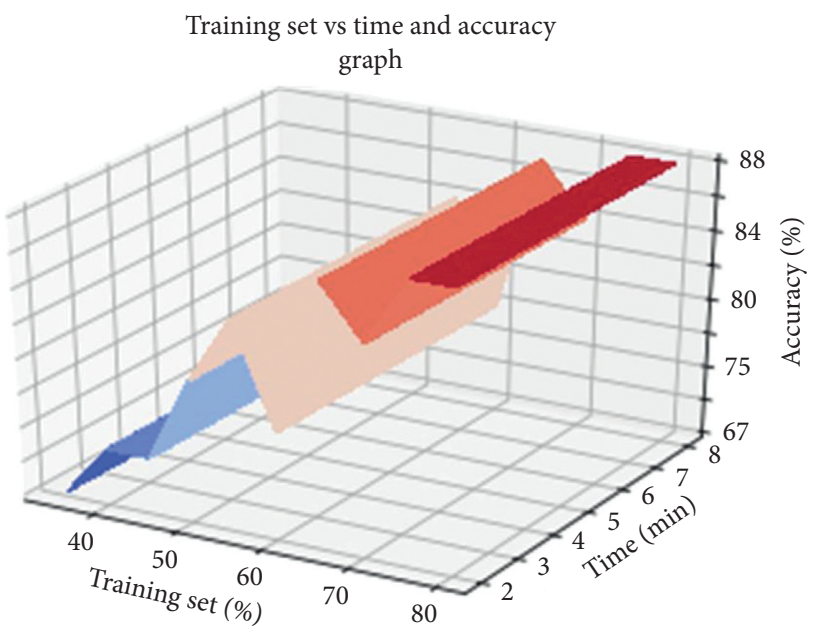

Figure 11: Recognition results of feedforward neural network.

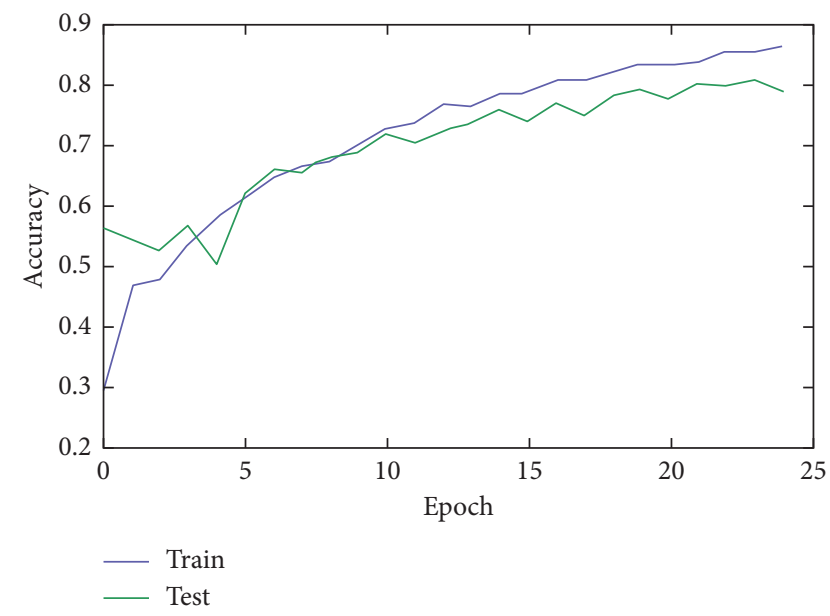

Figure 12: Proposed model accuracy.

\section{Results and Discussion}

The proposed IoT-based brain hemorrhage detection system presents a quality brain hemorrhage diagnosis device based on machine learning techniques. The machine learning techniques include support vector machine and feedforward neural network. This application provides a quality

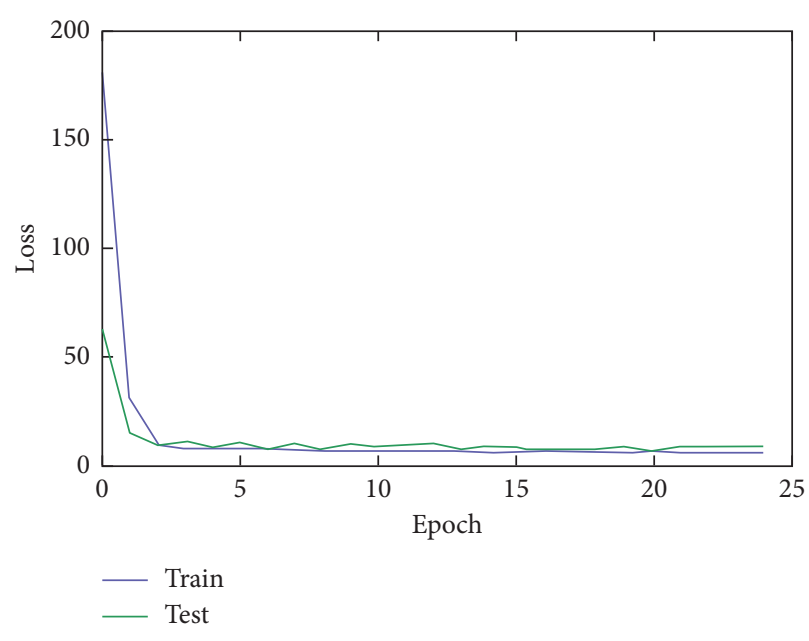

Figure 13: Proposed model loss.

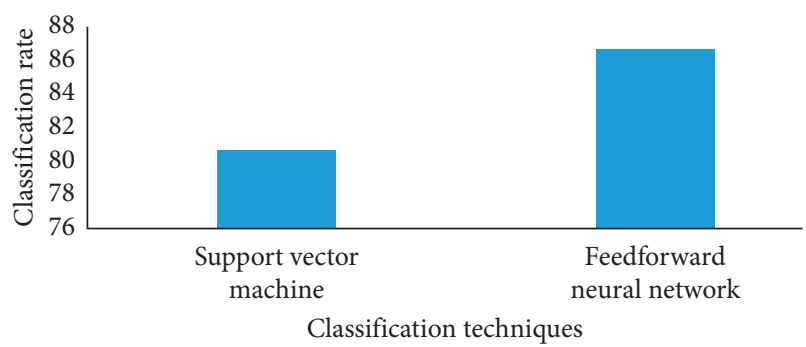

FIGURE 14: Classification results of the SVM and FNN techniques.

diagnosing facility for the brain hemorrhage patients. Regardless of the presence of a medical caretaker, a patient can visit the nearby hospital or even the one that has implemented this IoT-based application for diagnosing the brain hemorrhage. After diagnosing, this application has the ability to send the report to the patient and to the expert caretaker for suggesting the medicines accordingly. IoT devices like smart phones or laptops are handy in this case.

After applying the support vector machine and feedforward network to the brain hemorrhage dataset, an overall classification result of $80.67 \%$ and $86.7 \%$ is calculated, respectively, as shown in Figure 14.

From Figure 10, it is concluded that feedforward network outperforms in classifying the brain hemorrhage CT scan images based on different types of hemorrhage types. The SVM is a binary classifier and fails in resulting high classification rates for multiclass problems, while on the other side, FNN is prominent in this case.

\section{Conclusion}

This paper presents an IoT-based smart brain hemorrhage detection system using machine learning techniques. Automatic detection of brain hemorrhage is a very complex task, which mostly causes death or lifetime disabilities. To reduce these risks of causalities or lifetime disabilities and also to provide a quality treatment at door step, this paper has proposed a smart IoT application that accurately diagnoses the brain hemorrhage. This IoT-based application uses 
the support vector machine and feedforward neural network for classifying the brain hemorrhage types. Overall, accuracies of $80.67 \%$ and $86.7 \%$ are calculated for the SVM and FNN, respectively. Based on the classification results, it was concluded that the feedforward neural network gives prominent results compared to the support vector machine in a small interval of time. In future, the researchers may extend the proposed research work to cardiac disease detection using machine learning algorithms.

\section{Data Availability}

No data were used to support this study.

\section{Conflicts of Interest}

The authors declare no potential conflicts of interest with respect to the research, authorship, and/or publication of this article.

\section{Acknowledgments}

This work was sponsored in part by the National Natural Science Foundation of China (81602562).

\section{References}

[1] A. Redondi, M. Chirico, L. Borsani, M. Cesana, and M. Tagliasacchi, "An integrated system based on wireless sensor networks for patient monitoring, localization and tracking," Ad Hoc Networks, vol. 11, no. 1, pp. 39-53, 2013.

[2] S. Khan, "Waypoint navigation system implementation via a mobile robot using global positioning system (GPS) and global system for mobile communications (GSM) modems," International Journal of Computational Engineering Research (IJCER), vol. 3, no. 7, 2013.

[3] F. Ullah, A. H. Abdullah, O. Kaiwartya et al., "EETP-MAC: energy efficient traffic prioritization for medium access control in wireless body area networks," Telecommunication Systems, pp. 1-23, 2017.

[4] H. Asmat, F. Ullah, M. Zareei, A. Khan, and E. M. Mohamed, "Energy-efficient centrally controlled caching contents for information-centric internet of things," IEEE Access, vol. 8, pp. 126358-126369, 2020.

[5] H. Asmat, "ELC: edge linked caching for content updating in information-centric internet of things," Computer Communications, vol. 156, pp. 174-182, 2020.

[6] M. Ahmad, A. A. Ikram, I. Wahid, F. Ullah, A. Ahmad, and F. Alam Khan, "Optimized clustering in vehicular ad hoc networks based on honey bee and genetic algorithm for internet of things," Peer-to-Peer Networking and Applications, vol. 13, no. 2, pp. 532-547, 2020.

[7] M. D. Hssayeni, "Intracranial hemorrhage segmentation using deep convolutional model," 2019, https://arxiv.org/abs/ 1910.08643

[8] C. A. Taylor, J. M. Bell, M. J. Breiding, and L. Xu, "Traumatic brain injury-related emergency department visits, hospitalizations, and deaths-United States, 2007 and 2013," MMWR. Surveillance Summaries, vol. 66, no. 9, p. 1, 2017.

[9] C. J. van Asch, M. J. Luitse, G. J. Rinkel, I. van der Tweel, A. Algra, and C. J. Klijn, "Incidence, case fatality, and functional outcome of intracerebral haemorrhage over time, according to age, sex, and ethnic origin: a systematic review and meta-analysis," The Lancet Neurology, vol. 9, no. 2, pp. 167-176, 2010.

[10] S. Currie, N. Saleem, J. A. Straiton, J. Macmullen-Price, D. J. Warren, and I. J. Craven, "Imaging assessment of traumatic brain injury," Postgraduate Medical Journal, vol. 92, no. 1083, pp. 41-50, 2016.

[11] S. Nazir, "Internet of things for healthcare using effects of mobile computing: a systematic literature review," Wireless Communications and Mobile Computing, vol. 2019, Article ID 5931315, 2019.

[12] A. Onasanya and M. Elshakankiri, "IoT implementation for cancer care and business analytics/cloud services in healthcare systems," in Proceedings of The10th International Conference on Utility and Cloud Computing, Austin, TX, USA, December 2017.

[13] G. Cappon, "Wearable continuous glucose monitoring sensors: a revolution in diabetes treatment," Electronics, vol. 6, no. 3 , p. $65,2017$.

[14] F. Ullah, "Medium access control (MAC) for wireless body area network (WBAN): superframe structure, multiple access technique, taxonomy, and challenges," Human-centric Computing and Information Sciences, vol. 7, no. 1, p. 34, 2017.

[15] F. Ullah, "TraPy-MAC: traffic priority aware medium access control protocol for wireless body area network," Journal of Medical Systems, vol. 41, no. 6, p. 93, 2017.

[16] R. K. Kodali and B. S. Sarjerao, "MQTT based air quality monitoring," in Proceedings of the 2017 IEEE Region $10 \mathrm{Hu}$ manitarian Technology Conference (R10-HTC), Dhaka, Bangladesh, December 2017.

[17] G. Litjens, T. Kooi, B. E. Bejnordi et al., "A survey on deep learning in medical image analysis," Medical Image Analysis, vol. 42, pp. 60-88, 2017.

[18] A. Abdelaziz, "A machine learning model for predicting of chronic kidney disease based internet of things and cloud computing in smart cities," in Security in Smart Cities: Models, Applications, and Challenges, pp. 93-114, Springer, Berlin, Germany, 2019.

[19] S. S. Al-Majeed, I. S. Al-Mejibli, and J. Karam, "Home telehealth by internet of things (IoT)," in Proceedings of the 2015 IEEE 28th Canadian Conference on Electrical and Computer Engineering (CCECE), Halifax, Canada, May 2015.

[20] A. Dwivedi, G. Srivastava, S. Dhar, and R. Singh, "A decentralized privacy-preserving healthcare blockchain for iot," Sensors, vol. 19, no. 2, p. 326, 2019.

[21] F. Firouzi, Internet-of-Things and big data for smarter Healthcare: From Device to Architecture, Applications and Analytics, Elsevier, Amsterdam, Netherlands, 2018.

[22] M. Hassanalieragh, "Health monitoring and management using Internet-of-Things (IoT) sensing with cloud-based processing: opportunities and challenges," in Proceedings of the 2015 IEEE International Conference on Services Computing, New York, NY, USA, July 2015.

[23] S. Jabbar, "Semantic interoperability in heterogeneous IoT infrastructure for healthcare," Wireless Communications and Mobile Computing, vol. 2017, Article ID 9731806, 10 pages, 2017.

[24] J. Maktoubian and K. Ansari, "An IoT architecture for preventive maintenance of medical devices in healthcare organizations," Health and Technology, vol. 9, no. 3, pp. 233-243, 2019.

[25] A. A. Mutlag, M. K. Abd Ghani, N. Arunkumar, M. A. Mohammed, and O. Mohd, "Enabling technologies for fog computing in healthcare IoT systems," Future Generation Computer Systems, vol. 90, pp. 62-78, 2019. 
[26] P. M. Shakeel, "Maintaining security and privacy in health care system using learning based deep-Q-networks," Journal of Medical Systems, vol. 42, no. 10, p. 186, 2018.

[27] B. Farahani, F. Firouzi, V. Chang, M. Badaroglu, N. Constant, and K. Mankodiya, "Towards fog-driven IoT eHealth: promises and challenges of IoT in medicine and healthcare," Future Generation Computer Systems, vol. 78, pp. 659-676, 2018.

[28] I. Azimi, A. M. Rahmani, P. Liljeberg, and H. Tenhunen, "Internet of things for remote elderly monitoring: a study from user-centered perspective," Journal of Ambient Intelligence and Humanized Computing, vol. 8, no. 2, pp. 273-289, 2017.

[29] C. Cortes and V. Vapnik, "Support-vector networks," $M a$ chine Learning, vol. 20, no. 3, pp. 273-297, 1995.

[30] V. Vapnik and V. Vapnik, Statistical Learning Theory, pp. 156-160, Wiley, New York, NY, USA, 1998.

[31] H. Kaur and M. Kumar, "A comprehensive survey on word recognition for non-Indic and Indic scripts," Pattern Analysis and Applications, vol. 21, no. 4, pp. 897-929, 2018. 